\title{
Marcas discursivas de medicalización en anuncios publicitarios ${ }^{*}$
}

\author{
Adriana Margarita Plazas Salamanca*
}

Recibido: 15 de marzo de 2015

Evaluado: 9 de abril de 2015

Aceptado: 22 de abril de 2015

\section{ReSUMeN}

El presente artículo expone los elementos de construcción de la fecundación como problema biopolítico. A través de la revisión de los términos control natal y planificación familiar se observan las prácticas de medicalización que responden a políticas y programas de control demográfico. El objetivo reside en describir las marcas discursivas que evidencian las prácticas de medicalización presentes en las pautas publicitarias de la marca Norforms. La medicalización centra su interés en expandir sus acciones de control sobre la vida en el caso particular de los óvulos anticonceptivos, en el que se puede visibilizar la estrategia de creación de la enfermedad social y la promoción del tratamiento a través de las campañas de difusión de la higiene pública.

En el artículo se presentan los resultados del análisis de cuatro anuncios publicitarios de la marca Norforms publicadas en revistas que circulaban en Bogotá en 1964, 1972, 1991, 1998. Para el análisis discursivo se recurre a los conceptos de biopolítica y medicalización proporcionados por Michel Foucault a partir de los cuales se describen las dinámicas económicas y políticas de control poblacional.

Palabras clave: medicalización, biopolítica, publicidad, control natal, anticoncepción.

Este artículo de reflexión es producto de investigación que pertenece a la línea de análisis del discurso, adscrita al grupo Mente, lenguaje y sociedad del Departamento de Humanidades (clasificación A1 en Colciencias), Universidad Jorge Tadeo Lozano (Colombia). Cómo citar este artículo: Plazas Salamanca, A. (2015). Marcas discursivas de medicalización en anuncios publicitarios. Hallazgos, 12(24), 201-217 (doi: http://dx.doi.org/10.15332/ s1794-3841.2015.0024.11).

** Licenciada en Lingüística y Literatura, Universidad Distrital Francisco José de Caldas (Colombia); magíster en Lingüística Española, Instituto Caro y Cuervo (Colombia). Profesora de tiempo completo del Departamento de Humanidades, Universidad Jorge Tadeo Lozano (Colombia). Correo electrónico: adriana.plazas@utadeo.edu.co 


\section{Marks of discourse in advertisements medicalization}

\section{Abstract}

This paper presents the construction elements of fertilization as Received: March 15, 2015 biopolitical problem. Through reviewing the terms birth control Evaluated: April 9, 2015 and family planning practices medicalization that match policies and population observed control programs. The aim is to describe the discursive marks that show medicalization practices present in the advertising guidelines Norforms brand. The medicalization focuses its interest in expanding their control actions on life in the particular case of contraceptive ovules where you can visualize the strategy of creating social disease and treatment advocacy through the campaigns of public hygiene.

In the article, the results of the analysis of four mailers brand Norforms published in magazines circulating in Bogotá in the years presented 1964, 1972, 1991, and 1998. For discourse analysis of the concepts of biopolitics and medicalization recourse provided by Michel Foucault from which economic and political described dynamics of population control.

Keywords: Medicalization, biopolitics, advertising, birth control, contraception. 


\section{Marcas discursivas de medicalização em anúncios publicitários}

Recebido: 15 de março de 2015

Avaliado: 9 de abril de 2015 Aceito: 22 de abril de 2015

\section{Resumo}

Este artigo expõe os elementos de construção da fecundação como problema biopolítico. Através da revisão dos termos controle de natalidade y planificação familiar observam-se as práticas de medicalização que respondem a politicas y programas de controle demográfico. O objetivo radica em descrever as marcas discursivas que evidenciam as práticas de medicalização presentes nas pautas publicitárias da marca Norforms. A medicalização centra seu interesse na expansão de suas ações de controle sobre a vida, no caso particular dos óvulos anticonceptivos, nesta pode-se fazer visível a estratégia de criação da doença social y a promoção do tratamento através das campanhas de difusão da higiene pública.

No artigo apresentam-se os resultados da análise de quatro anúncios publicitários da marca Norforms publicados em revistas que estiveram em circulação em Bogotá durante 1964, 1972, 1991 e 1998. Para a análise discursiva recorre-se aos conceitos de biopolítica e medicalização propostos pelo Michel Foucault a partir dos quais descrevem-se as dinâmicas econômicas e políticas do controle populacional.

Palavras-chave: medicalização, biopolítica, publicidade, controle da natalidade, anticoncepção. 


\section{INTRODUCCIÓN}

El control natal responde a prácticas discursivas relacionadas con un doble discurso. Por una parte, con el instaurado por Thomas Malthus y su teoría demográfica, según la cual la población crecería más rápido que el suministro de comida. Este argumento se adoptó para pensar en un control natal como política económica que permita evitar la escasez de medios de subsistencia. Por otra parte, está el discurso de John Stuart Mill según el cual el control natal se hace necesario para asegurar una calidad de vida de la población. Al pensar en cuántos hijos se pueden sostener para llevar una vida digna, es necesario el control de la fecundación, el cual hace ineludible el uso de los anticonceptivos (Schoijet, 2007; Gordon, 1973;Tone, 1996). Al respecto, Michel Foucault permite aproximarse al asunto a partir de la noción de biopolítica, la cual trabaja con la población como problema biológico y de poder, y se entiende como una tecnología que se instala en los procesos de la proporción de los nacimientos y los decesos, la tasa de reproducción, la fecundidad de la población, en conexión con todo un conjunto de problemas económicos y políticos (Foucault, 1976, 2007).

La importancia que cobra el cuerpo y su control es uno de los problemas que estudia Michel Foucault y que sirve como eje teórico para realizar una reflexión acerca de los mecanismos que utiliza el discurso publicitario para convertir en necesidad, de la población femenina, la utilización de productos como parte de un circuito en el que los Estados, los Gobiernos y las autoridades sanitarias, implementan políticas y programas sanitarios de control de la sexualidad (Foucault, 1991, 1981).
Con la separación del sexo y la procreación, el dispositivo biopolítico de control y vigilancia sobre el cuerpo determina y administra las zonas erógenas, el placer y el género, así como los ciclos de fertilidad y en este caso particular, la anticoncepción. Para acercarme a los textos publicitarios que he escogido para mi análisis centraré la atención en el cambio de sentido y de rótulo del fenómeno de la contracepción trabajando con el cambio del significado de los términos en un lapso corto.

Los discursos que circulaban en la primera mitad del siglo XX estaban dirigidos en su preferencia hacia la mujer, insistiendo permanentemente en el temor del embarazo y todos los problemas relacionados con la repetida concepción, los abortos e incluso la muerte. Hacia 1930 en Norteamérica se inicia una campaña de control natal que surgió de la crisis de la Gran Depresión que afectó económicamente a la población mundial, la cual incitó al cambio de prácticas de control natal. La industria farmacéutica apoyada en el discurso científico puso en marcha una lucrativa empresa que estaba centrada en alimentar el deseo de las personas por reducir el tamaño de sus familias ${ }^{1}$ (Tone, 1996, 2000).

Así, la anticoncepción permite ver prácticas discursivas dinámicas que han ido cambiando de acuerdo con los espacios de experiencia en los que estos conceptos se van formulando. Siguiendo a Reinhart Koselleck (1993), "un concepto [...] no solo indica unidades de acción: también las acuña y crea. No es solo un indicador, sino también

\footnotetext{
Las empresas farmacéuticas jugaron un papel importante en este capítulo del control de la natalidad. Por una parte, fabricaban, distribuían y vendían una gran variedad de productos entre las mujeres y, por otra parte, evadían las normas legales que prohibían la distribución y venta de anticonceptivos (Tone, 1996, p. 486).
} 
un factor de grupos políticos o sociales" (p. 206); así es posible rastrear el concepto de anticoncepción en la dinámica de los procesos económicos y políticos que rodean al término y de ese modo ver cómo se mantienen los sentidos o se enriquecen en esta pequeña temporalidad. Entre 1964 y 1998² se presentan cuatro rótulos para referirse a la anticoncepción: control natal, planificación familiar, atención materna infantil y salud sexual y reproductiva.

\section{Control natal}

El concepto control natal ha tenido una larga existencia, según la historiadora Linda Gordon (citada por Mauricio Schoijet, 2007). Gordon plantea que hubo por lo menos tres fases diferentes del movimiento de control de la natalidad. A la primera fase la podríamos llamar utópica o romántica; a la segunda, radical, y a la última, burguesa. La primera inició en la segunda mitad del siglo XIX, y era referida bajo el lema de maternidad voluntaria a través de la propuesta irreal de la abstinencia. La segunda fase, llamada radical, comenzó hacia 1915 en Norteamérica, y surgió como un movimiento en favor del control de la natalidad, con intervención de militantes socialistas y feministas. Tuvo una participación masiva de mujeres proletarias, y fue dirigido por la enfermera Margaret Sanger, famosa por abrir en 1916 una clínica en Brooklyn. Sanger fue líder en el movimiento de control de natalidad durante varias décadas a pesar de su polémica actuación, dado que participó en prácticas de eugenesia en contradicción a su discurso

2 El corpus del presente artículo toma cuatro anuncios publicitarios publicados en diferentes años y en diferentes revistas: Vanidades Continental, 1964, Selecciones, 1972, Revista femenina (sin nombre), 1991, Revista Shock, 1998. inicial en defensa de la salud y la economía familiar. La tercera fase corresponde a la participación de mujeres ricas, conservadoras anglosajonas y protestantes que participaron como activistas de los derechos de las mujeres, la educación sexual y el voto femenino. Se destaca la figura de María Ware Dennett, quien fundó la Voluntary Parenthood League y escribió un famoso texto sobre la educación sexual y métodos anticonceptivos. De igual modo, gestionó el apoyo público en miras a derogar leyes que restringían la información sobre el control de la natalidad ${ }^{3}$. El concepto de control natal se instala, como lo podemos constatar, con la idea ligada a un discurso de higiene; es decir que con el empleo de elementos de uso externo y métodos de barrera es posible dominar la fecundación y obtener protección, seguridad, tranquilidad y un buen matrimonio (Tone, 2000). En la imagen que promocionaba la ducha sanitaria Lysol, se puede apreciar ese discurso dirigido a las amas de casa, pletórico de la retórica de la desinfección. El texto les informaba que sus vaginas estaban sucias y olorosas, lo que ocasionaba la repulsión de sus maridos. La solución a sus problemas maritales era entonces la limpieza utilizando las duchas vaginales ${ }^{4}$.

La publicidad de la época elaboraba mensajes cifrados en el mito de la doncella medrosa y despreciada por el varón dominante. En este tipo de anuncios se encuentran dos discursos: por una parte, el de la higiene; por otra parte, está el de la contracepción.

3 Ver: http://en.wikipedia.org/wiki/Mary_Dennett

4 La publicidad centrada en discursos de estigma y vergüenza afianzados por el desconocimiento provocó el uso masivo de estos productos químicos que provocaban serias enfermedades y además no prevenían el embarazo (Gerson Institute, 2014). 
Figura 1. Publicidad Lysol.

\section{"PLEASE, DAVE...PLEASE DON'T LET ME BE LOCKED OUT FROM YOU!"}

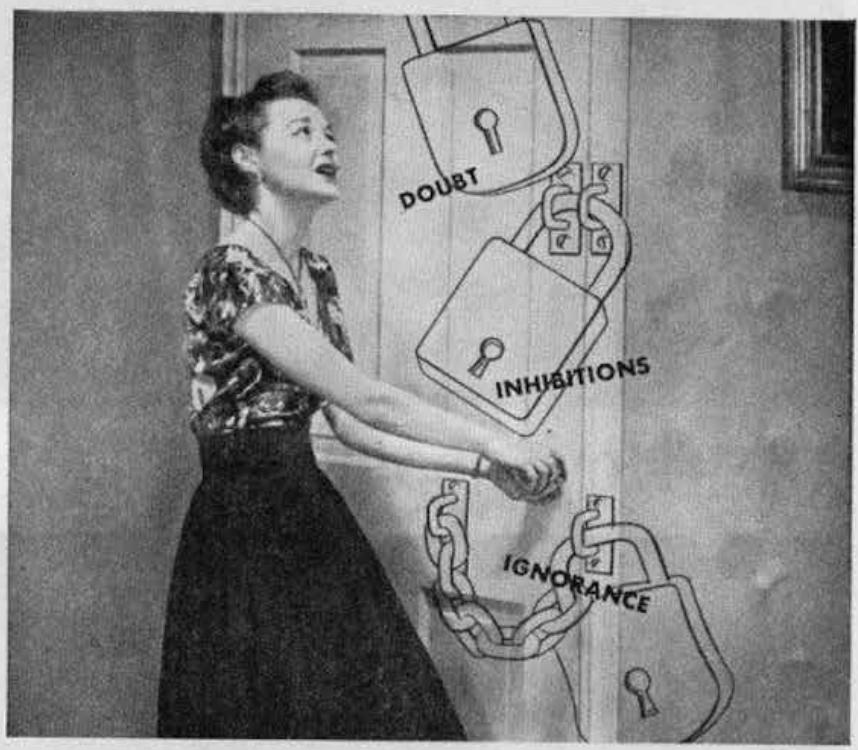

Offen a wife fails to realize that doubts due to one intimate neglect shut her out from happy married love

A man marries a woman because he loves her. So instead of blaming him if married love begins to cool, she should question herself. Is she truly trying to keep her husband and herseif eager, happy married lovers? One most effective way to safeguard her dainty feminine allure is by practicing complete feminine hygiene as provided by vaginal douches with a scientifically correct preparation like "Lysol." So easy a way to banish the misgivings that often keep married lovers apart.

\section{Germs destroyed swiffly}

"Lysol" has amazing, proved power to kill germ-life on contact ... truly cleanses the vaginal canal even in the presence of mucous matter. Thus "Lysol" acts in a way that makeshifts like soap, salt or soda never can.

Appealing daintiness is assured, be. cause the very source of objectionable odors is eliminated.

\section{Use whenever needed!}

Yet gentle, non-caustic "Lysol" will not harm delicate tissue. Simple directions give correct douching solution. Many doctors advise their patients to douche regularly with "Lysol" brand disinfectant, just to insure feminine daintiness alone, and to use it as often as necessary. No greasy aftereffect.

For feminine hygiene, three times more women use "Lysol" than any other liquid preparation. No other is more reliable. You, too, can rely on "Lysol" to help proteet your married happiness ... keep you desirable!
For complete Feminine Hygiene rely on...

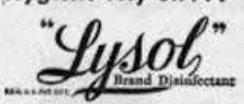

\section{A Concentrafed} Germ-Killer

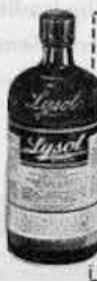

\section{NEWI,..,FEMININE HYGIENE FACTS}

FREEI New booklet of information by leading gynecological authority. Mail coupon to Lehn \& Fink, 192 Bloomfield Avenue, Bloomfield, N. J.

Name

Sureed

Ciny-

Product og Taken \& Wink

Fuente: Gerson Institute (2014) 
Ya se venía utilizando la expresión "mata gérmenes" (germicida) como sustituto de la prevención del embarazo (espermicida).

\section{Planificación familiar}

Después de la Segunda Guerra Mundial la preocupación general se centró en el crecimiento de la población, el cual fue adoptado como un problema y una empresa política y socioeconómica acogida internacionalmente. Como prueba de ello se crearon diferentes instituciones y proyectos de financiamiento para planes de Control natal entre los que se cuentan la Federación Internacional de Planificación Familiar (International Planned Parenthood Federation [IPPF] $)^{5}$, la Fundación Ford y la Fundación Rockefeller, Fondo de Población de las Naciones Unidas (FNUAP).

Aunque la preocupación era mundial y los países europeos lo pusieron en sus agendas como tema prioritario, fue Estados Unidos quien impulso políticas específicas y planes estratégicos hacia Latinoamérica. Linda Gordon, citada por Felitti, afirma que:

En los años cuarenta había surgido allí un importarte movimiento de family planning, cuyo objetivo fundacional había sido promover el bienestar familiar como precondición para conservar el capitalismo y sus promesas de libertad y prosperidad. No se trataba de cuestionar el modelo de familia imperante y con ella el sistema social en general, como habían hecho muchas militantes

5 Este organismo, que reunía a un conjunto de asociaciones nacionales autónomas, promovía la implementación de programas de planificación familiar a gran escala y a través de sus agencias, brindaba asistencia sanitaria y educativa a las parejas que lo solicitasen (Felitti, 2008). feministas y socialistas de principios de siglo, sino de crear condiciones más auspiciosas para la llegada de la prole (2009, p. 57).

De este modo, se puso en marcha en el continente la política de planificación familiar haciendo uso de numerosas estrategias, conferencias, asambleas y seminarios, asesoría técnica, investigación y apoyo financiero, con el fin de proyectar el desarrollo como una estrategia con la cual se podría erradicar la pobreza, favorecer el crecimiento económico y evitar la expansión del comunismo (Morales Borrero, 2010, p. 57).

El discurso de higiene continuase verbaliza a través de las expresiones problemas maritales, problemas embarazosos. En el primero la joven esposa es instruida en el secreto; en el segundo anuncio se inicia la vida marital, el discurso de la intimidad, de las respuestas a las complicaciones que conlleva un embarazo no planificado.

\section{ANTICONCEPCIÓN: ¿RESPUESTA A UN PROBLEMA MÉdico O POLÍtICO?}

La definición más difundida del término medicalización la proporciona el médico Jugal Kishore ${ }^{6}$, especialista en salud pública y epidemiología: "la forma en que el ámbito de la medicina moderna se ha expandido hacia muchos problemas que antes no estaban considerados como entidades médicas" (citado por Martínez, 2008). La medicalización así entendida permite ver el cambio de objeto de estudio, en el cual, en una perspectiva anterior a la llamada medicina moderna, no entraban como enfermedades ciertos asun-

6 Jugal Kishore escribió A dictionary of Public Health. 
Figura 2. Publicidad Norforms.

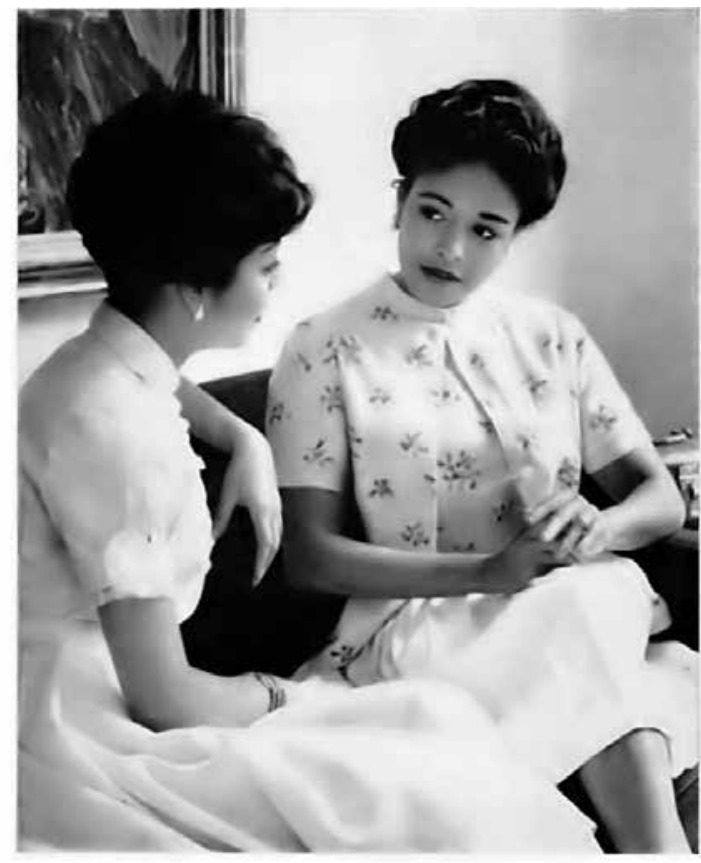

\section{Married women are sharing this secret}

...the new, easier, surer protection for those most intimate marriage problems

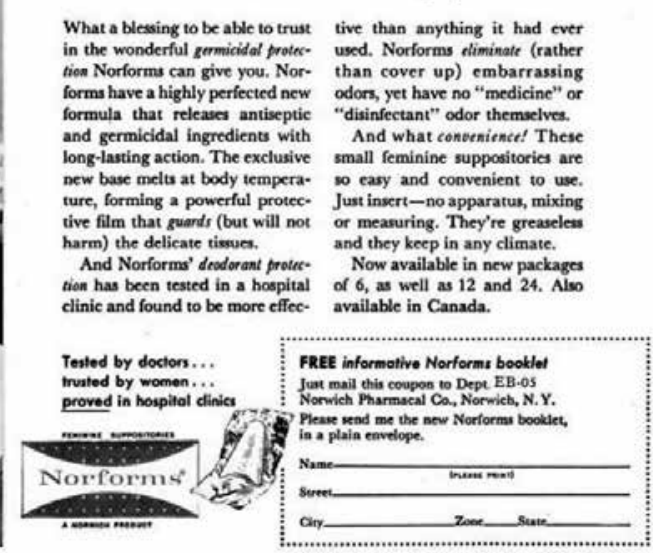

Fuente: Ebony (mayo, 1960).

tos cotidianos como los ciclos femeninos referidos a la menstruación, la concepción, la lactancia y la menopausia. Ahora desde una nueva óptica se consideran estos como patológicos y además pasan a formar parte del mercado de la enfermedad.

Este anuncio trabaja sobre la divulgación y el compartir secretos y experiencias del cuidado de la llamada higiene marital, el cual es el discurso de la planificación familiar. Ebony es una revista para el mercado afroamericano.

El escritor y médico Venancio Martínez declara que "[...] por la vía de la medicalización se transforman en enfermedades simples dolencias, pequeñas molestias o inquietudes de la más variada índole. [...] la medicalización logra hacer de "la salud" y del sistema sanitario un artículo de consumo más" (2008, p. 19). En este sentido, salud y enfermedad, riesgo y prevención, salud pública y epidemiologia pasan a ser parte de un mismo campo. Es tan amplio el campo de acción de la medicalización que ahora se describen como enfermedades todos los procesos importantes del ser humano, las etapas de desarrollo y crecimiento, la vejez, o fenómenos que son producto de la sociedad como la pobreza, la desdicha, la delincuencia.

El trabajo de la medicina según Michel Foucault se centra en torno a la higiene pública y su dinámica de trabajo: “[...] se llevará a cabo a través de organismos que coordinan y centralizan las curas médicas, hacen circular información, normalizan el saber, hacen 
Figura 3. Publicidad Norforms

\section{New answer for the intimate, embarrassing problems married women face.}

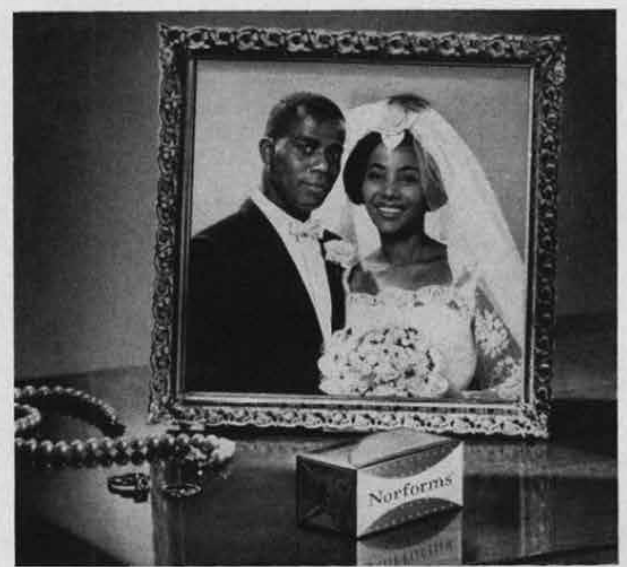

Tiny, germicidal Norforms protects you more effectively than douching.

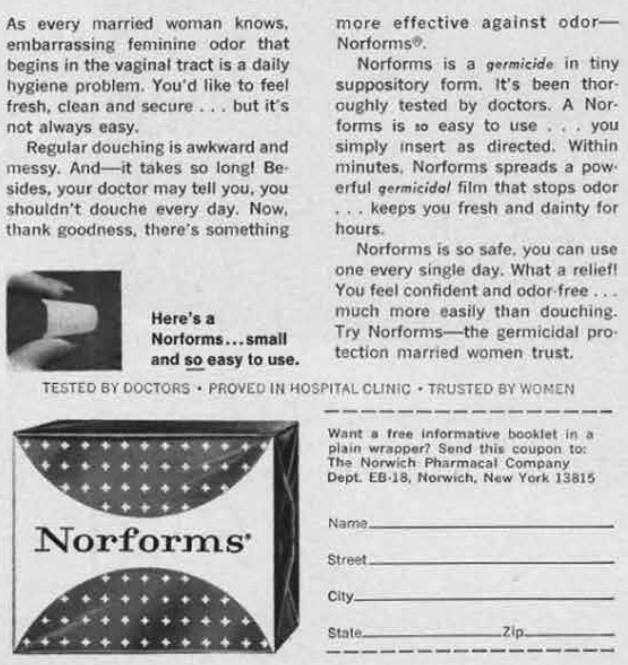

Fuente: Ebony (1968). campañas para difundir la higiene y trabajan por la medicalización de la población. Los problemas fundamentales que esa medicina deberá afrontar son los de la reproducción, de la natalidad y de la morbilidad" (1976, p. 197). Desde este punto de vista se puede analizar la anticoncepción como un fenómeno de medicalización de la sexualidad como una práctica política y económica que tiende a resolver el viejo argumento malthusiano.

\section{Análisis de anuncios PUBLICITARIOS NORFORMS}

Hemos realizado un marco global desde el que se puede visualizar la complejidad del asunto que nos convoca, esto es la publicidad de productos de higiene íntima. Durante el siglo XX se creó el mito de que uno de los métodos anticonceptivos efectivos era el del lavado vaginal en el sobrentendido de que al aplicar jabones era posible matar el espermatozoide; así se creó un discurso cuyo mecanismo iba del tabú sexual al malentendido morfológico. Ya que no se podía hablar de sexo, sexualidad, coito, embarazo, ni contracepción, se creó una jerga sobre la que los textos publicitarios hacían circular la información a la población femenina.

En el siguiente segmento se realizará el análisis de estas marcas discursivas desde las cuales se construye la enfermedad y se da la solución. A partir de una descripción de una enfermedad social se propone el tratamiento; esto es, se insta al consumo del producto anunciado.

La publicidad de la empresa Norwich Pharmaceuticals, fabricante de los óvulos Norforms, me ha llamado la atención en 
cuanto a la amplia y reiterada manera de divulgar su producto por más de sesenta años. Durante los años treinta en Norteamérica se amplió la demanda por productos de control de la natalidad por parte de muchas mujeres; así ni las instituciones de salud ni las farmacéuticas podían cubrir las necesidades de una nación entera que reclamaba tanto información sobre métodos anticonceptivos, como atención. La empresa Norwich, como tantas otras en el mercado, convirtió a Norforms en "la marca más popular de supositorios vaginales en el país desplegando un argumento para justificar su política publicitaria" la cual consiste en utilizar una idea ya establecida para vender su producto utilizando las expectativas creadas por el anuncio (Tone, 1996, p. 494$)^{7}$. Es en este orden de ideas que podemos analizar el anuncio de 1964 aparecido en la revista Vanidades Continental.

\section{Noticia importante. Para mujeres casadas, 1964}

Con el título sugerente "Noticia importante. Para mujeres casadas" se puede ver la marca de un discurso de la llamada higiene del matrimonio, la cual está mezclada con un texto seudocientífico para lograr el efecto deseado: el consumo del producto.

7 Andrea Tone en su artículo afirma que para la época los supositorios Norforms "se anunciaban exclusivamente como la higiene femenina, un término que el vicepresidente de la compañía Webster Stofer admitió había convertido en sinónimo de la anticoncepción en la mente de muchas mujeres. De todos modos, Stofer insistió, Norforms no fue vendido como control de la natalidad. Al preguntarle por qué la empresa no cambiaba su eslogan de marketing para evitar malentendidos, Stofer expresó su pesar de que era 'demasiado tarde' para anunciar supositorios como cualquier otra cosa. 'El término está estrechamente asociado con Norforms' Stofer sostuvo. Y de todos modos, tenemos nuestra propia definición de la misma'"' (1996, p. 494).
Figura 4. Publicidad Norforms.

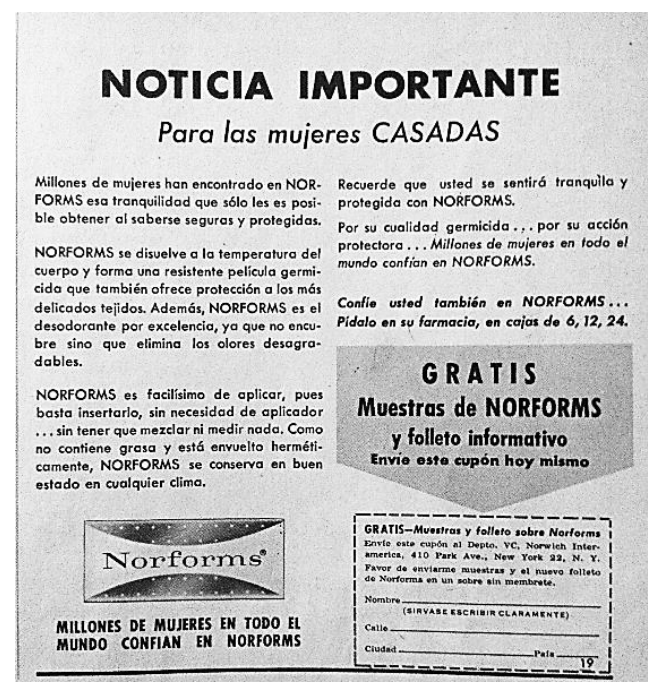

Fuente: Vanidades Continental (1964).

El cupón de envío de muestras y folleto tiene una línea de solicitud "favor de enviarme muestras y el nuevo folleto de Norforms en un sobre sin membrete". Así se asegura la privacidad y la discreción de la comunicación, quizás por el tabú reinante en torno a la sexualidad. La dirección que aparece es la de los laboratorios en Nueva York.

El problema superficial que se plantea es "el olor desagradable" que puede ser eliminado gracias a la acción efectiva del producto. He aquí la estrategia de la creación del problema: el olor. ¿Por qué es importante y qué presuponía? La sexualidad, la genitalidad, el lugar del encuentro de los fluidos y por lo tanto el lugar donde existía el peligro de la fecundación significaban un problema de demografía.

Para esta época existían en el mercado muchos métodos anticonceptivos (la píldora, el condón, las jaleas, los diafragmas y el supositorio vaginal); sin embargo, también circulaban discursos opuestos que provenían de diferentes ámbitos sociales. Por una 
parte, la Iglesia y los conservadores persistirán con gran fuerza en la oposición radical a la utilización de métodos anticonceptivos; por otro lado, la presión que ejercían los Estados y los Gobiernos.

Con respecto a las políticas que en Colombia se venían estableciendo, María Carolina Morales Barrero plantea en su investigación:

En el país, el manejo del problema del crecimiento de la población durante la posguerra se llevó a cabo con la influencia de las políticas norteamericanas y sus desarrollos en las políticas dirigidas a la mujer. Del control natal se pasó a la planificación de la familia; y después, a la atención materno-infantil, hasta llegar a las políticas de salud sexual y reproductiva. Pasaron diez años, entre 1964 y1974, para pasar de la planificación familiar a las políticas de salud sexual y reproductiva (2010, p. 56).

Como se ve en la figura1, la publicidad vende la idea de mujer dominada y desdeñada, la cual se continúa en la publicidad para Latinoamérica, aunque de manera mucho más cauta. "[...]NORFORMS es el desodorante por excelencia, ya que no encubre sino que elimina los olores desagradables". La eliminación del olor es el eufemismo creado para denominar la anticoncepción (Tone, 1996). De ahí se entiende la serie de sustantivos que acompañan el anuncio: tranquilidad, seguridad, protección y confianza, todos ellos son marcas lingüísticas relacionadas con el discurso de la higiene. Por otra parte, está el argumento del número de usuarias "Millones de mujeres en todo el mundo confían en Norforms". Si muchas lo usan es porque es bueno y seguro. Para Colombia la venta de este producto tenía el ingrediente adicional de la participación en un circuito de correspondencia con el laboratorio productor en Nueva York; esto es, el prestigio de ser contactada para recibir el producto que viene de fuera y además recibir la información deseada sobre su uso.

\section{El cuerpo de la mujer debería venir con instrucciones, 1972}

El anuncio aparecido en la revista Selecciones denota un discurso sexista atravesado por una lección seudocientífica elaborada en un largo texto de 12 párrafos, en los que se cosifica el cuerpo femenino, se problematiza el olor y se da una solución: el uso de los supositorios. En realidad lo que hace este anuncio es continuar con la elaboración discursiva de los años sesenta.

Cabe anotar que la dirección de correspondencia es de los laboratorios Norwich colombiana con un apartado aéreo en Bogotá.

Es patente la evocación que hace del mito de Venus que permite hacer una lectura aún más elaborada relacionada con el amor sexual, la enfermedad por el contacto sexual.

Al utilizar de nuevo el problema del olor como huella del sexo: "Las glándulas del sudor en las regiones de la axila y púbica producen el peor tipo de olor. Este tipo de sudor, intensificado por la estimulación sexual o emocional, es atrapado por el pelo aumentando de esta manera la posibilidad de olor". En la figura 5, la joven cubre con sus manos las zonas mencionadas: la axila y el pubis. De nuevo se trabaja con el discurso ambiguo que ofrece un desodorante y un anticonceptivo: "Con Norforms, correctamente empleado, usted no tendrá ningún problema embarazoso". El concepto de control 
natal permanece, se ha instalado, gracias a la repetibilidad del discurso de la higiene con la cual se puede evitar la concepción.

En Colombia durante el Gobierno de Lleras Restrepo la política de planificación familiar

Figura 5. Publicidad Norforms.

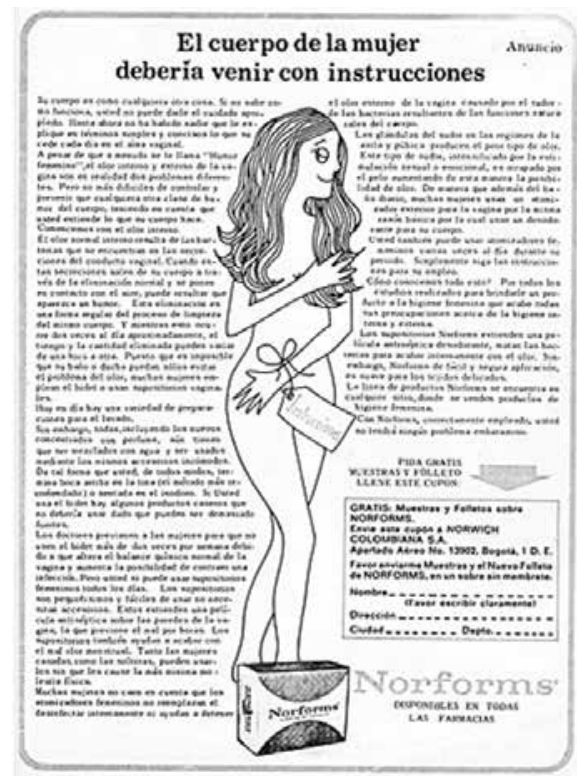

Fuente: Selecciones(1972).

Figura 6.

El nacimiento de Venus.

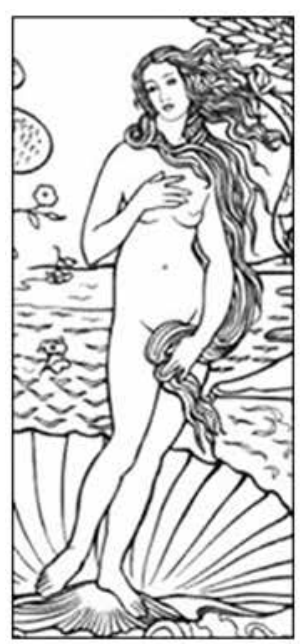

fue reemplazada por un programa especial dirigido a proteger la salud materno-infantil y para tal efecto emite la

[...] ley de paternidad responsable y contribuir a su materialización creando el Instituto Colombiano de Bienestar Familiar, ICBF[...]dicha ley además de impulsar una idea tradicional de familia, pretendía neutralizar a la Iglesia Católica y responder a las preocupaciones sobre el reconocimiento de los hijos naturales, la nutrición, el cuidado del menor y de la familia [...] Finalmente, durante el gobierno de Lleras Restrepo, la planificación familiar se delegó a un actor privado denominado Profamilia. Esta era la mejor manera de pasar inadvertida, y además facilitó la importación directa de los anticonceptivos. La entidad se instaló con financiación norteamericana, con apoyo de un sector de los médicos, del Estado y de la industria (Morales Borrero, 2010, p. 58).

Se puede observar en el anuncio el tránsito del argumento del número de usuarias al de la disponibilidad en "todas las farmacias" "Norforms se encuentra en cualquier sitio, donde venden productos de higiene femenina", así como la posibilidad de uso: "Tanto las mujeres casadas, como las solteras, pueden usarlos sin que les cause la más mínima molestia física."

\section{0 minutos antes...para que no haya problemas después, 1991}

Los jóvenes se han convertido en el blanco publicitario atendiendo a discursos sobre sexualidad, placer y responsabilidad. La redacción de estos dos anuncios publicados en los años noventa llama la atención por 
Figura 7. Publicidad Norforms (1991)

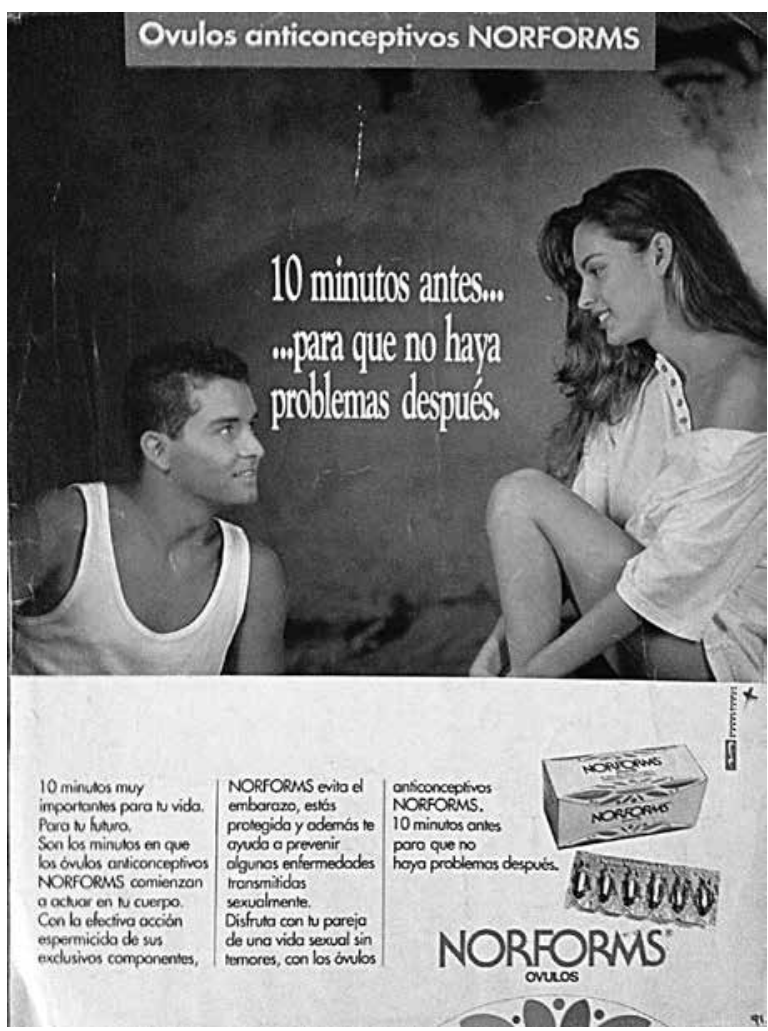

Figura 8. Publicidad Norforms.

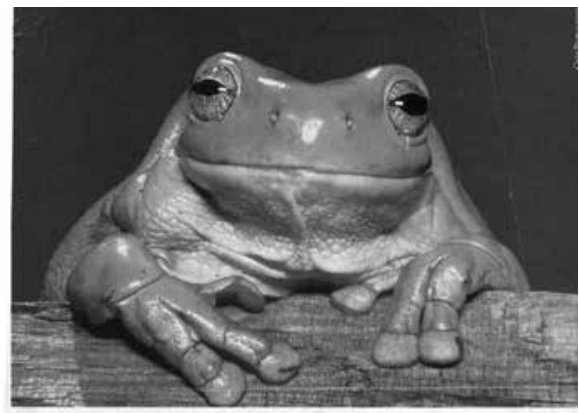

Si Piensas Acostarte con tu Principe Azul ...NO TE DUERMAS.

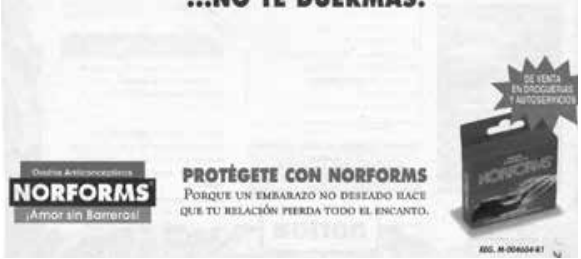

Fuente: Shock (1998). 
varias razones. En el primero se hace uso del lenguaje directo explícito en que los óvulos son anticonceptivos, "evitan el embarazo" y además "previene[n] enfermedades de transmisión sexual". La foto que acompaña al anuncio hace referencia al placer en pareja. El público al que va dirigido es distinto, ya no es la mujer solitaria sufriendo por el problema, ahora es un problema que se comparte: "disfruta con tu pareja de una vida sin temores".

En Colombia las diferentes políticas del Ministerio de Educación Nacional continuaban con los programas de comportamiento y salud instaurados en los años setenta que se impartían en los grados $5^{\circ}$ y $6^{\circ}$ del nivel secundario con énfasis en anatomía y biología, los cuales resultaron ser un fracaso ya que informaban pero no formaban ${ }^{8}$. Luego se inició el discurso de la educación sexual basada en los derechos y la opción de una sexualidad plena y responsable ${ }^{9}$.

\section{¡Amor sin barreras!, 1998}

Es en este marco donde puede explicar el anuncio de lafigura8 ¡Amor sin Barreras!, el cual está diseñado sobre el esquema "problema-solución-producto"10. Inicia el texto exponiendo la sexualidad como el problema y para tal efecto emplea una sugestiva foto de un sapo venenoso, la cual ocupa casi la mitad del anuncio. Además, utiliza la estructura del argumento condicional "si -entonces"

8 "El embarazo adolescente se ubicó en primer plano; la tasa de fecundidad reportada por la Encuesta Nacional de Demografía y Salud (ENDS), en 1995, fue de 89 por 1000, y la proporción de embarazos en mujeres adolescentes alcanzó el 19\% de la población femenina en ese grupo de edad, en contraste con la tasa reportada para 1990, que fue de 70 por mil" (Morales Borrero, 2010, p. 109).

9 (Morales Borrero, 2010, p. 154).

10 En realidad los siete anuncios trabajados en el presente artículo trabajan sobre este mismo esquema de diseño. el cual está cifrado en el código de los cuentos de hadas: "Si piensas acostarte con tu príncipe azul... no te duermas". La ambigüedad que ofrece la expresión duermas también es importante, ya que gira en el sentido de tener relaciones sexuales, por una parte, y de descuido, por otra. Como consecuencia, presenta la solución con lo enunciado "protégeme con Norforms" . El razonamiento se completa con la información redactada otra vez con un conector causal al inicio del enunciado: "Porque un embarazo no deseado hace que tu relación pierda todo el encanto". El texto completo se podría reducir al siguiente esquema: peligro-sexo-protección-Norforms. En él se sintetizan aspectos importantes en el tema de la medicalización: por una parte, la sexualidad como un enemigo mortal, un problema que debe ser combatido con la prevención del embarazo y por lo tanto con el uso del producto en cuestión. Al igual que el anterior texto publicitario, se utiliza el acercamiento al receptor a través del tuteo como forma de tratamiento que intenta despertar confianza y solidaridad.

Como se ha podido constatar, el papel que juega la publicidad en el circuito del biopoder es el de mecanismo de divulgación de políticas y programas sanitarios. En ese sentido, hacen circular la información de un producto que ha sido convertido en necesidad y normalizan el saber científico, valiéndose de este como argumento de autoridad para realizar campañas con el fin de difundir el discurso de higiene, protección y prevención a la población.

\section{Discusión}

En la realización de esta indagación pude describir las marcas discursivas presentes 
en los anuncios de este famoso producto de uso cotidiano entre las mujeres de diferentes generaciones. En el proceso pude constatar que el dispositivo de sexualidad que se dinamiza en el discurso de la anticoncepción, el cual se puede distinguir en todo el proceso de la medicalización-desde la invención de las enfermedades hasta la construcción de un discurso convincente que tiende a exagerar las dimensiones de la enfermedades-, utiliza una lógica persuasiva como estrategia de marketing para promocionar y reproducir el discurso de la enfermedad y de los productos que la combaten.

He revisado la publicidad de casi sesenta años sobre los productos anticonceptivos y en la búsqueda de los óvulos Norforms ingresé a la página en español ${ }^{11}$. En esta, sorpresivamente, encontré que los discursos de los anuncios impresos de 1991 y 1998 se oponían a la información que allí se presenta. En el anuncio aparece un aviso que dice:

"ADVERTENCIA: LOS SUPOSITORIOS DESODORANTES NORFORMS NO SON UN ANTICONCEPTIVO.

Consulte a su Médico antes de usar este producto si se encuentra embarazada".

¿Por qué si en la página web oficial de Norforms se divulga como producto desodorante, en Colombia en las revistas y páginas web se publicita como un artículo anticonceptivo?

Los estudios médicos afirman que este tipo de óvulos tienen una baja efectividad en la prevención del embarazo; sin embargo, llama la atención que en los impresos

11 Ver: http://www.norforms.com/espanol/ mencionados se haya publicado explícitamente como óvulos anticonceptivos. Esta publicidad engañosa responde a las prácticas de las industrias farmacéuticas en las que la máxima es crear necesidades a toda costa.

Así de devela en este sencillo análisis que por más de un siglo lo que se inició como una reflexión demográfica, económica y sociopolítica pasó a ser una estrategia de control de la población que llega a invadir los asuntos más íntimos de la vida humana.

\section{REFERENCIAS}

Bacaj, A. (septiembre, 2014). Please, Dave... plase don't let me be locked outa from you. Recuperado de https://gerson.org/gerpress/the-dirty-secrets-of-feminine-hygiene-products/

Boyer, A. (agosto, 2012). Biopolítica y filosofía feminista. Revista de Estudios Sociales(43), 131-138. Recuperado de http:// dx.doi.Org/10.7440/res43.2012.11

Briggs, L. (2010). Reproductive Technology: Of Labor and Markets. Feminist Studies, 36 (2,RE-INVENTING MOTHERS), 359-374. Recuperado el 20 de enero de 2015, de http://www.jstor.org/ stable/27919105

Espósito, R. (2006). Bíos. Biopolitica y filosofía. Buenos Aires: Amorrortu.

Felitti, K. (2008). La "explosión demográfica" y la planificación familiar a debate: Instituciones, discusiones y propuestas del centro y la periferia. Revista Escuela de Historia [online], 7(2). Recuperado el 1 de diciembre de 2014, de http://www. scielo.org.ar/scielo.php?script=sci_arttext\&pid=S1669-90412008000200003\&1$\mathrm{ng}=\mathrm{es \& nrm}=\mathrm{iso}>$ 
Felitti, K. (septiembre, 2009). Derechos reproductivos y políticas demográfica en América latina. Íconos. Revista de Ciencias Sociales.[on line](35), 55-66. Recuperado el 1 de diciembre de 2014, de http://www. redalyc.org/articulo.oa?id $=50911906005$

Foucault, M. (1976). Genealogía del racismo. Buenos Aires: Altamira.

Foucault, M. (1977). Historia de la medicalización. Educación médica y salud, 11(1), 3-25. Recuperado el 20 de enero de 2015, de http://hist.library.paho.org/Spanish/ EMS/4839.pdf

Foucault, M. (1981). El nacimiento de la clínica. Una arqueología de la mirada médica. México: Fondo de Cultura Económica.

Foucault, M. (1991). Historia de la sexualidad 1: la voluntad de saber. México, D. F.: Fondo de Cultura Económica.

Foucault, M. (2007). Nacimiento de la biopolítica: cursos en el Collège de France. México, D. F.: Fondo de Cultura Económica.

Gerson Institute. (septiembre 2014). The Dirty Secrets of Feminine Hygiene Products. Recuperado el 30 de noviembre de 2014, de gerson.org: http://gerson.org/ gerpress/about-us/

Gordon, L. (1973). Voluntary Motherhood; The Beginnings of Feminist Birth Control Ideas in the United States. Recuperado el 20 de enero de 2015, de http://www.jstor.org/stable/1566477

Koselleck, R. (1993). Semántica del cambio histórico de la experiencia. En Futuro pasado.Para una semántica de los tiempos históricos. (pp. 205-250). Barcelona: Paidós.

López-Ibor Alino, J. M. (1981). Biblioteca básica de la educación sexual. El libro de los anticonceptivos. Madrid: Ediciones Uve.

Martínez Suárez, V. (2008). Salud pública, medicalización de la vida y educación.
En V. Martínez Suárez, C. Ponte, R. Huertas, R. Cabo, C. Amorós y A. González (Eds.), Salud y ciudadanía. Teoría y práctica de la innovación [en línea] (pp. 18-27). Gijón: Centro del Profesorado y de Recursos de Gijón. Recuperado el 2 de 12 de 2014, de 85.152.37.8/recursos/ 2008/SaludyCiudadania.pdf

Morales Borrero, M. (2010). Jóvenes, sexualidad y politicas. Salud sexual y reproductiva en Colombia (1992-2005). Bogotá: Universidad Nacional de Colombia. Recuperado el 27 de noviembre de 2014, de http://www.bdigital.unal.edu. co/3403/4/Jovenes_Sexualidad_y_Politica.pdf

Norforms. (mayo, 1960). Married women are sharing this secret. Ebony, $X V(7)$, 15. Recuperado de https://books.google.com.co/books?id=QoVz2rpp-skC \&printsec $=$ frontcover\&dq=ebony $+\mathrm{ma}-$ gazine $+1960+$ may \&hl=en\&sa $=X \&$ ei $=\mathrm{i}-$ I10VY2uDoScsAWs84LYAQ\&ved=$0 \mathrm{CB} 8 \mathrm{Q} 6 \mathrm{AEwAQ}$ $\mathrm{v}=$ onepage \&q=norforms\&f=false

Norforms. (1964). Noticia Importante para las mujeres casadas. Vanidades Continental.

Norforms. (enero, 1968). Embarrassing Problems Married Women Face. Ebony, XXIII(3), 10. Recuperado el 22 de noviembre de 2014, de https://books.google.com.co/books?id=2dkDAAAAM $\mathrm{BAJ} \&$ printsec $=$ frontcover\&source $=\mathrm{gbs}$ ge_summary_r\&cad $=0 \# \mathrm{v}=$ onepage $\&$ $\mathrm{q}=$ norforms $\& \mathrm{f}=$ false

Norforms. (1972). El cuerpo de la mujer debería venir con instrucciones. Selecciones.

Norforms. (1991). 10 minutos antes... para que no haya problemas después. 
Norforms. (1998). Si piensas acostarte con tu princicpe azul... no te duermas. Revista Shock.

Rodríguez Zoya, P. G. (julio, 2010). La medicalización como estrategia biopolítica. A Parte Rei. Revista de Filosofía, (70), 1-26. Recuperado el 20 de enero de 2015, de http://serbal.pntic.mec.es/ cmunoz11/ zoya70.pdf

Sánchez Torres, F. (1991). Ciencia y reproducción humana. Bogotá: Universidad Nacional de Colombia.

Schoijet, M. (octubre-diciembre, 2007). El control de la natalidad:un esbozo de historia. Papeles de Población, 13(54), 115-161.

Tejeda González, J. L. (2011). Biopolitica, control y dominación. Recuperado el 20 de enero de 2015, de http://www.scielo.org. $\mathrm{mx} / \mathrm{pdf} / \mathrm{espiral} / \mathrm{v} 18 \mathrm{n} 52 / \mathrm{v} 18 \mathrm{n} 52 \mathrm{a} 3 . \mathrm{pdf}$

Tietze, C. (julio1965). History of Contraceptive Methods.Recuperado el 20 de enero de 2015, de http://www.jstor.org/ stable/3811880

Tone, A. (1996). Contraceptive Consumers: Gender and the Political Economy of Birth Control in the 1930s. Journal of Social History, 29(3), 485-506.

Tone, A. (septiembre, 2000). Black Market Birth Control:Contraceptive Enterpreneurship and Criminality in the Gilded Age. The Journal of American History, 87(2), 435-459. Recuperado el 20 de enero de 2015, de http://www.jstor.org/ stable/2568759 